\title{
Predictive impact of absolute lymphocyte counts for progression-free survival in human epidermal growth factor receptor 2-positive advanced breast cancer treated with pertuzumab and trastuzumab plus eribulin or nab-paclitaxel
}

Kazuhiro Araki ${ }^{1,2}$, Yoshinori Ito ${ }^{2}$, Ippei Fukada ${ }^{2}$, Kokoro Kobayashi², Yoshimasa Miyagawa ${ }^{1}$, Michiko Imamura', Ayako Kira', Yuichi Takatsuka ${ }^{1}$, Chiyomi Egawa ${ }^{3}$, Hirofumi Suwa ${ }^{4}$, Shinji Ohno ${ }^{2}$ and Yasuo Miyoshi ${ }^{1 *}$

\begin{abstract}
s
Background: Although peripheral blood-based parameters (PBBPs) are reported as prognostic indicators in patients with breast cancers, their utility has not been studied in human epidermal growth factor receptor 2 (HER2)-positive advanced breast cancer (ABC). Tumor-infiltrating lymphocytes (TILs) might be a predictive factor in patients with HER2-positive ABC treated with pertuzumab and trastuzumab (PT) plus docetaxel. We aimed to evaluate whether PBBPs could have predictive value in HER2-positive ABC treated with pertuzumab and trastuzumab (PT) combined with eribulin (ERI) or nab-paclitaxel (Nab-PTX).

Methods: Data from 51 patients included in two single-arm, phase II trials were included in this retrospectiveprospective study; the ERI + PT $(N=30)$ and Nab-PTX + PT $(N=21)$ combinations were registered under clinical trials number UMIN000012375 and UMIN000006838, respectively. We assessed PBBPs using prospectively collected data and investigated the association with progression-free survival (PFS); we evaluated absolute lymphocyte count (ALC), neutrophil to lymphocyte ratio (NLR) and platelet to lymphocyte ratio (PLR). The cutoff values for ALC, NLR, and PLR were set at 1000 or 1500 cells/ $\mu \mathrm{L}, 2$, and 250, respectively.

Results: PFS was significantly improved in patients with ALC $\geq 1500 / \mu \mathrm{L}$ compared to those with ALC 1000-, $<1500 /$ $\mu \mathrm{L}$ or ALC $<1000 / \mu \mathrm{L}(P=0.0106)$. High baseline ALC was significantly associated with improved PFS $(\geq 1500 / \mu \mathrm{L}$; hazard ratio [HR]: 0.3715; 95\% confidence interval [Cl]: 0.1735-0.7955; $P=0.0108)$. In contrast, improved PFS was not significantly associated with NLR or PLR. Improved PFS in patients with ALC $\geq 1500 / \mu L$ was observed irrespective of visceral metastasis or chemotherapy regimen.

(Continued on next page)
\end{abstract}

\footnotetext{
* Correspondence: ymiyoshi@hyo-med.ac.jp

${ }^{1}$ Hyogo College of Medicine, Department of Surgery, Division of Breast and

Endocrine Surgery, 1-1 Mukogawa, Nishinomiya, Hyogo 663-8501, Japan

Full list of author information is available at the end of the article
}

(c) The Author(s). 2018 Open Access This article is distributed under the terms of the Creative Commons Attribution 4.0 International License (http://creativecommons.org/licenses/by/4.0/), which permits unrestricted use, distribution, and reproduction in any medium, provided you give appropriate credit to the original author(s) and the source, provide a link to the Creative Commons license, and indicate if changes were made. The Creative Commons Public Domain Dedication waiver (http://creativecommons.org/publicdomain/zero/1.0/) applies to the data made available in this article, unless otherwise stated. 


\begin{abstract}
(Continued from previous page)
Conclusions: Our results showed that baseline ALC was a predictive factor for PFS in HER2-positive ABC treated with PT irrespective of combined chemotherapy regimen. Anti-tumor effects might be mediated not only by the tumor microenvironment, but also by systemic peripheral circulating lymphocytes. Baseline systemic parameters such as peripheral lymphocyte count might be beneficial in addition to disease extent for predicting the efficacy of PT treatment.
\end{abstract}

Trial registration: UMIN000012375, registration date: 21NOV2013, and UMIN000006838, registration date: 6DEC2011.

Keywords: Absolute lymphocyte counts, Predictive biomarker, Pertuzumab, Trastuzumab, HER2-positive ABC, Host immunity

\section{Background}

During the past decade, advanced human epidermal growth factor receptor 2 (HER2)-targeted therapies have significantly improved outcomes for patients with HER2-positive advanced breast cancer (ABC) [1]. Clinical evaluations of pertuzumab and trastuzumab (PT) in combination with different chemotherapy regimens in the CLEOPATRA trial and our previous study have highlighted clinical benefits $[2,3]$.

Pertuzumab and trastuzumab are humanized monoclonal anti-HER2 antibodies with complementary proposed mechanisms of action. Both antibodies work through the disruption of HER2 dimerization with HER3 and other epidermal growth factor receptors (EGFRs), leading to the inhibition of HER2 signaling [4]. Anti-HER2 antibodies are thought to mediate tumor regression not only by interrupting oncogenic signaling, but also by inducing antibody-dependent cell-mediated cytotoxicity (ADCC) [4]. ADCC can induce innate immune system responses via Fcy receptors. These responses are sustained by natural killer (NK) cells; concomitantly, adaptive immunity involving tumor-infiltrating lymphocytes (TILs) develops in response to a specific antigen, which is sustained by $\mathrm{T}$ and B lymphocytes [5]. The association of TILs with clinical outcomes suggests a significant role for antitumor immunity in HER2-positive ABC [6].

Immune responses against cancer cells might involve a balance between tumor inhibitory mechanisms induced by intrinsic immunity and the ability of tumor cells to evade immune surveillance [7]. During cancer immunoediting phases (i.e., elimination, equilibrium, and escape), activated effector cytotoxic $\mathrm{T}$ lymphocytes can migrate to the tumor and infiltrate the tumor microenvironment $[7,8]$. It has been reported that circulating peripheral lymphocytes and neutrophils can migrate towards a tumor site in a directed manner along the humoral factors, such as a chemoattractant [9]. A recent study demonstrated that circulating tumor-specific $\mathrm{T}$ lymphocytes related to neoantigens can be isolated from the peripheral blood of patients with melanoma [10]. These data may indicate that blood-based parameters (PBBPs) reflect a local immune reaction against cancer cells. In line with such a mechanism, the absolute lymphocyte count (ALC), neutrophil-to-lymphocyte ratio (NLR), and platelet-to-lymphocyte ratio (PLR) are reported to be immune-related prognostic factors for patients with malignancy [11, 12]. In addition, relationships between low levels of NLR and improved response to neoadjuvant chemotherapy have been reported for patients with breast cancer [13-15]. Therefore, PBBPs seem to affect treatment efficacy for $\mathrm{ABC}$ treated with chemotherapy and anti-HER2 therapy; however, this topic has yet to be fully studied.

In the present study, we aimed to explore whether PBBPs are related to improved progression-free survival (PFS) in patients with HER2-positive ABC treated with HER2-targeted PT combined with eribulin (ERI) or nab-paclitaxel (Nab-PTX).

\section{Methods}

\section{Study design and participants \\ Patients}

Data from 51 patients included in two single-arm, phase 2 trials were included in this retrospective-prospective study (ERI + TP $[N=30]$ and Nab-PTX + TP $[N=21])$ registered under University Hospital Medical Information Network (UMIN)000012375 and UMIN000006838, respectively, and approved by the ethics committee of The Cancer Institute Hospital of the Japanese Foundation for Cancer Research (\#2013-1096 and \#2017-1076) and the Hyogo College of Medicine (No. 1061 and No. 2720), respectively.

Drug dosing schedule in two prospective phase II studies In the UMIN000006838 study, Nab-PTX $260 \mathrm{mg} / \mathrm{m}^{2}$ was administered intravenously over $30 \mathrm{~min}$ every 3 weeks. Two-step dose reductions to $220 \mathrm{mg} / \mathrm{m}^{2}$ and $180 \mathrm{mg} / \mathrm{m}^{2}$ were allowed before discontinuation was considered. Details of the dosing schedule in the UMIN000012375 study were published in our previous report [3]. Dose reductions were allowed for both drugs but not for PT, whereas dose interruptions were allowed for all drugs. PT was administered according to a standard procedure as published previously [3]. 


\section{Evaluation of predictive factors}

Data for PBBPs at baseline were obtained on the same day and just before the start of the first cycle of chemotherapy administration. No patient was administered granulocyte-colony stimulating factor at the last cycle of prior chemotherapy. Based on our study protocol, registration criteria required an interval of more than 3 weeks since a patient's last chemotherapy to minimize the influence of prior cytotoxic treatment. We evaluated ALC, NLR, and PLR data at baseline; the cutoff values for ALC, NLR, and PLR were set at 1000 or 1500 cells/ $\mu \mathrm{L}, 2$, and 150 , respectively. Cutoff values for both NLR and PLR were defined as the median value of each parameter. The cutoff value for ALC was based on the previous study [16].

\section{Statistics}

\section{PFS analysis}

PFS was defined as the time from treatment initiation to the date of disease progression or death; it was censored at the last adequate tumor assessment date before the cutoff date if no PFS event was observed prior to that time. PFS distributions were estimated using the Kaplan-Meier method.

\section{Univariate and multivariate analyses}

To evaluate the relationship between PFS and all clinical factors, univariate Cox proportional hazard regression models were used. Candidate predictive factors with a $P$-value less than 0.05 by univariate analysis were selected for evaluation by multivariate analysis. The value of ALC in terms of PFS was evaluated using a forest plot for each subgroup, dichotomized as $<1500$ and $\geq 1500$ cells $/ \mu \mathrm{L}$.

We tested associations between ALC and baseline clinicopathological characteristics using the Wilcoxon rank sum test for continuous variables with two groups for age and previous chemotherapy regimens, and Fisher's exact test for other categorical variables, as shown in Tables 1 and 3. All statistical analyses were performed using JMP Pro 13 (SAS Institute Inc., Cary, NC, USA) and GraphPad Prism 6 (GraphPad Software, Inc., La Jolla, CA, USA). $P$ values of less than 0.05 were considered statistically significant.

\section{Results}

\section{Demographics and baseline clinical characteristics}

Table 1 shows a summary of the baseline clinical characteristics of patients from both studies and the differences between the ERI and Nab-PTX groups. A total of 51 patients (median age, 58 years) were enrolled; of these, 49 patients (96\%) had favorable Eastern Cooperative Oncology Group Performance Status (ECOG-PS) scores of 0 or 1. Patients in the Nab-PTX group had significantly better ECOG-PS than those in the ERI group $(P<0.0001)$. Forty patients $(78 \%)$ had HER2 overexpression (3+) confirmed by immunohistochemistry (IHC), and 11 patients (22\%) with IHC 2+ status were confirmed to be HER2 positive by fluorescence in situ hybridization. Twenty-three cases (45\%) were estrogen receptor (ER) positive, whereas 11 patients (22\%) were progesterone receptor (PgR) positive. Visceral metastases and non-visceral metastases were reported in 19 (37\%) and 32 (63\%) patients, respectively. Patients with visceral metastases (50\%) were significantly more in the ERI group than in the Nab-PTX group $(19 \%, P=0.0389)$. Patients received a median of 3 (range, 1-10) prior treatments. There was no significant difference between groups in any parameters other than PS and metastatic sites.

\section{Efficacy}

Anti-tumor activity

All patients in the full-analysis set were evaluable for overall response rate (ORR). Investigator assessments identified 4 patients with complete response (8\%), while 15 patients $(29 \%)$ achieved partial response. The ORR was $37 \%$, the disease control rate was $78 \%(N=40)$, and the clinical benefit rate was $55 \%(N=28)$. The median PFS was 301 days (95\% confidence interval [CI]: 212568) based on Kaplan-Meier estimates. There was no significant difference in PFS between ERI and Nab-PTX (hazard ratio [HR]: 1.593, 95\% CI [0.75103.378], $P=0.2249$ ).

\section{Univariate and multivariate analyses of PFS according to the baseline clinical factors}

Table 2 provides a summary of univariate and multivariate analyses of PFS according to baseline clinical characteristics. Univariate analysis showed that patients aged $<58$ years, those with ALC $\geq 1000 / \mu \mathrm{L}$ and ALC $\geq 1500 / \mu \mathrm{L}$, and those with less than 3 lines of prior treatment had better PFS (Table 2). Both NLR and PLR showed a trend toward statistical significance (Table 2). ER and PgR positivity, HER2-3+, presence of visceral metastases, and treatment during study were not significant predictors of PFS (Table 2).

In the multivariate analysis, only ALC $\geq 1500 / \mu \mathrm{L}$ (HR: 0.296; 95\% CI, 0.098-0794; $P=0.0150$ ) was found to be correlated with PFS (Table 2). PFS was also significantly longer in patients with ALC $\geq 1500 / \mu \mathrm{L}$ compared with $1000-1500 / \mu \mathrm{L}$ or $<1000 / \mu \mathrm{L}$ (median PFS: not reached versus 363 versus 238 days, respectively; $P=0.0106$; Fig. 1). An exploratory analysis (Fig. 2) of the HRs associated with ALC $\geq 1500 / \mu \mathrm{L}$ or $<1500 / \mu \mathrm{L}$ consistently favored PFS in patients with ALC $\geq 1500 / \mu \mathrm{L}$, irrespective of age ( $<58$ and $\geq 58$ years), HER2 levels $(3+$ and $2+)$, metastatic sites (visceral or non-visceral), 
Table 1 Demographics and baseline characteristics of patients

\begin{tabular}{|c|c|c|c|c|c|}
\hline & & Eribulin $(N=30)$ & Nab-paclitaxel $(N=21)$ & Total $(N=51)$ & $P$ value \\
\hline Median age & (range) & $58(31-76)$ yo & 58 (32-77) yo & $58(31-77)$ & 0.5524 \\
\hline \multirow[t]{3}{*}{ Performance status } & 0 & $10(33 \%)$ & $20(95 \%)$ & $30(59 \%)$ & $<0.0001$ \\
\hline & 1 & $18(60 \%)$ & $1(5 \%)$ & $19(37 \%)$ & \\
\hline & 2 & $2(7 \%)$ & 0 & $2(4 \%)$ & \\
\hline \multirow[t]{2}{*}{ HER2 immunohistochemical staining } & $3+$ & $25(83 \%)$ & $15(71 \%)$ & $40(78 \%)$ & 0.327 \\
\hline & $2+$ (with FISH amplification) & $5(17 \%)$ & $6(19 \%)$ & $11(22 \%)$ & \\
\hline \multirow[t]{2}{*}{ Estrogen receptor } & positive & $14(47 \%)$ & $9(43 \%)$ & $23(45 \%)$ & 1.000 \\
\hline & negative & $16(53 \%)$ & $12(57 \%)$ & $28(55 \%)$ & \\
\hline \multirow[t]{2}{*}{ Progesterone receptor } & positive & $5(17 \%)$ & $6(19 \%)$ & $11(22 \%)$ & 0.327 \\
\hline & negative & $25(83 \%)$ & $15(71 \%)$ & $40(78 \%)$ & \\
\hline \multirow[t]{2}{*}{ Metastatic sites } & visceral & $15(50 \%)$ & $4(19 \%)$ & $19(37 \%)$ & 0.0389 \\
\hline & non-visceral & $15(50 \%)$ & $17(81 \%)$ & $32(63 \%)$ & \\
\hline Previous chemotherapy regimens, median & (range) & $3.5(1-9)$ & $1(1-10)$ & $3(1-10)$ & 0.0579 \\
\hline
\end{tabular}

and treatment (ERI or Nab-PTX), except in cases that were PgR-positive.

A comparison of baseline characteristics based on ALC $\geq 1500 / \mu \mathrm{L}$ is shown in Table 3. There was no significant difference in the relationship between ALC levels and any factors, including metastatic site, number of prior treatments, or regimens.

\section{Discussion}

In this prospective-retrospective evaluation of PBBPs in baseline clinical data, we identified ALC as a predictive factor for PFS in patients with HER2-positive ABC. Additionally, high ALC at baseline was significantly associated with improved PFS in HER2-positive ABC treated with either ERI or Nab-PTX in combination with PT. To the best of our knowledge, this study is the first to analyze predictive factors associated with ALC in
HER2-positive $A B C$. For treatment of $A B C$, predictive factors related to systemic immune response are yet to be considered in clinical practice.

Usually, somatic mutations in cancer cells lead to the production of altered proteins that are recognized as antigens by the innate immune system via major histocompatibility complex class I; consequently, TILs inhibit tumor progression [17]. Anti-HER2 antibodies also mediate anticancer effects in part via the induction of ADCC by opsonizing cancer cells that are recognized by the innate immune system [18]. Anti-HER2 antibodies not only neutralize the trophic function of HER2, but they also elicit an initial NK-mediated ADCC response that is presumably followed by a cytotoxic $\mathrm{T}$ lymphocyte-dependent adaptive immune repose directed against breast cancer associated antigens [18]. The effect of pertuzumab in antitumor immunity is

Table 2 Univariate and multivariate analyses of progression-free survival

\begin{tabular}{|c|c|c|c|c|c|c|c|c|}
\hline \multirow[b]{2}{*}{ Parameter } & \multicolumn{2}{|c|}{ Number of patients } & \multicolumn{3}{|c|}{ Univariate } & \multicolumn{3}{|c|}{ Multivariate } \\
\hline & Yes & No & $\overline{\mathrm{HR}}$ & $95 \% \mathrm{Cls}$ & $P$ value & $\overline{\mathrm{HR}}$ & $95 \% \mathrm{Cls}$ & $P$ value \\
\hline Age $<58$ yo & $23 / 51$ & $28 / 51$ & 2.440 & $1.120-5.316$ & 0.0248 & 0.315 & $0.074-1.467$ & 0.1367 \\
\hline Estrogen receptor positive (vs. negative) & $23 / 51$ & $28 / 51$ & 1.198 & $0.555-2.588$ & 0.6448 & & & \\
\hline Progesterone receptor positive (vs. negative) & $11 / 51$ & $40 / 51$ & 1.177 & $0.456-3.040$ & 0.7361 & & & \\
\hline HER2 3+ & $40 / 51$ & $11 / 51$ & 1.421 & $0.558-3.618$ & 0.5431 & & & \\
\hline Non-visceral metastases (vs. visceral) & $32 / 51$ & $19 / 51$ & 0.926 & $0.429-1.996$ & 0.8441 & & & \\
\hline Eribulin (vs. Nab-PTX) & $30 / 51$ & $21 / 30$ & 1.593 & $0.751-3.378$ & 0.2249 & & & \\
\hline Prior treatment $<3$ line & $30 / 51$ & $21 / 30$ & 0.401 & $0.185-0.870$ & 0.0208 & 0.528 & $0.234-1.157$ & 0.1106 \\
\hline Absolute lymphocyte count $\geq 1000 / u L$ & $37 / 51$ & $14 / 51$ & 0.308 & $0.133-0.714$ & 0.0061 & 0.735 & $0.284-1.770$ & 0.4977 \\
\hline Absolute lymphocyte count $\geq 1500 /$ L & $19 / 51$ & $32 / 51$ & 0.372 & $0.174-0.796$ & 0.0108 & 0.296 & $0.098-0.794$ & 0.0150 \\
\hline Neutrophil to lymphocyte ratio > 2 & $31 / 51$ & $20 / 51$ & 2.109 & $0.985-4.516$ & 0.0548 & & & \\
\hline Platelet to lymphocyte ratio > 150 & $31 / 51$ & $20 / 51$ & 2.038 & $0.962-4.318$ & 0.0632 & & & \\
\hline
\end{tabular}




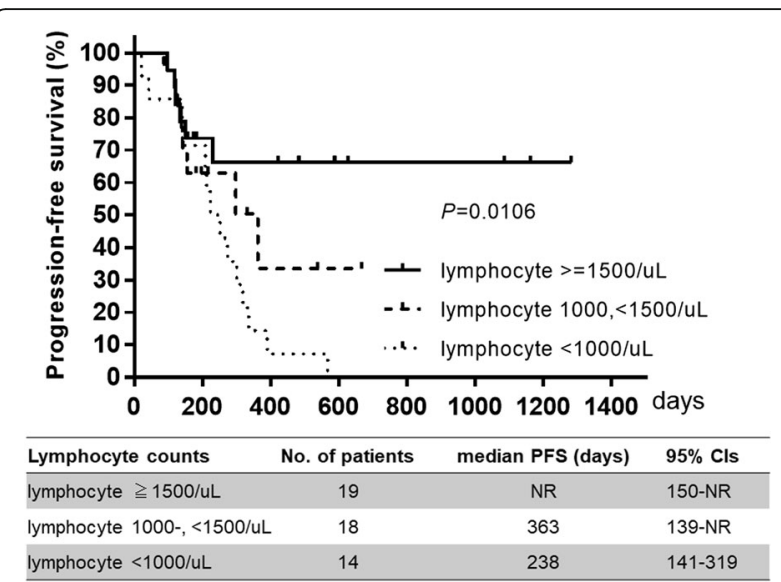

Fig. 1 Comparison of progression-free survival in patients according to absolute lymphocyte counts. Progression-free survival (PFS) was significantly longer in patients with absolute lymphocyte counts $(\mathrm{ALC}) \geq 1500 / \mu \mathrm{L}(P=0.0106)$. Solid lines indicate $\mathrm{ALC} \geq 1500 / \mu \mathrm{L}$, broken lines indicate ALC 1000-, <1500/ $\mu \mathrm{L}$, and dotted lines indicate ALC $<1000 / \mu \mathrm{L}$. Time (days) indicates the duration from the start of treatment to the occurrence of events. NR, not reached

still unknown. In a subgroup analysis of the CLEOPATRA trial, patients with a high abundance of TILs $(>20 \%)$ had better PFS than those with low TILs $(\leq 20 \%)$ in the PT group, but not in the trastuzumab group [6]. These data might indicate that preexisting immune responses enhance treatment efficacy, which could be boosted by combination therapy with conventional chemotherapy and PT [19]. Although prognostic markers including NLR and PLR have been evaluated in several malignant diseases [12], our study demonstrated that ALC is superior to NLR and PLR for predicting improved PFS in $\mathrm{ABC}$ patients treated with conventional chemotherapy combined with PT. High ALC may indicate enhanced immunity in tumors; alternatively, ALC may be a potential biomarker of host immunity. Although the detailed underlying mechanism of prolonged PFS in patients with ALC $\geq 1500 / \mu \mathrm{L}$ is currently unknown, it is likely that these results reflect the synergistic activity of PT acting alongside the host immune response.

Our results showed that the favorable PFS in patients with ALC $\geq 1500 / \mu \mathrm{L}$ was prominent in the ER- and PgRsubgroups. It is well established that TILs are not frequently observed in ER+/HER2- breast cancers [20]; thus, the prognostic significance of TILs is not recognized in these cases [20]. Even among HER2+ breast cancers, the frequency of TILs was significantly lower in ER+ cases compared with ER- cases [6]. These data indicate that anticancer immunity is not dominant in ER+ cases. Thus, ALC may be a less effective predictor in ER+ and PgR+ cases compared to ER- and PgR- cases.

Both ERI and Nab-PTX could be classified as microtubule inhibitors with differential mechanisms of action [21]. Both drugs can cause G2/M cell cycle arrest due to disruption of mitotic spindles, resulting in apoptotic cell death after prolonged mitotic blockage [22]. In the current study, a favorable PFS in patients with ALC $\geq 1500 / \mu \mathrm{L}$ was observed, irrespective of a combination treatment with ERI or Nab-PTX. Because chemotherapies differ with regard to immunogenicity (e.g., immunogenic [doxorubicin or cyclophosphamide] or non-immunogenic [dacarbazine] [21]), it is unclear whether relationships exist between PFS and ALC in

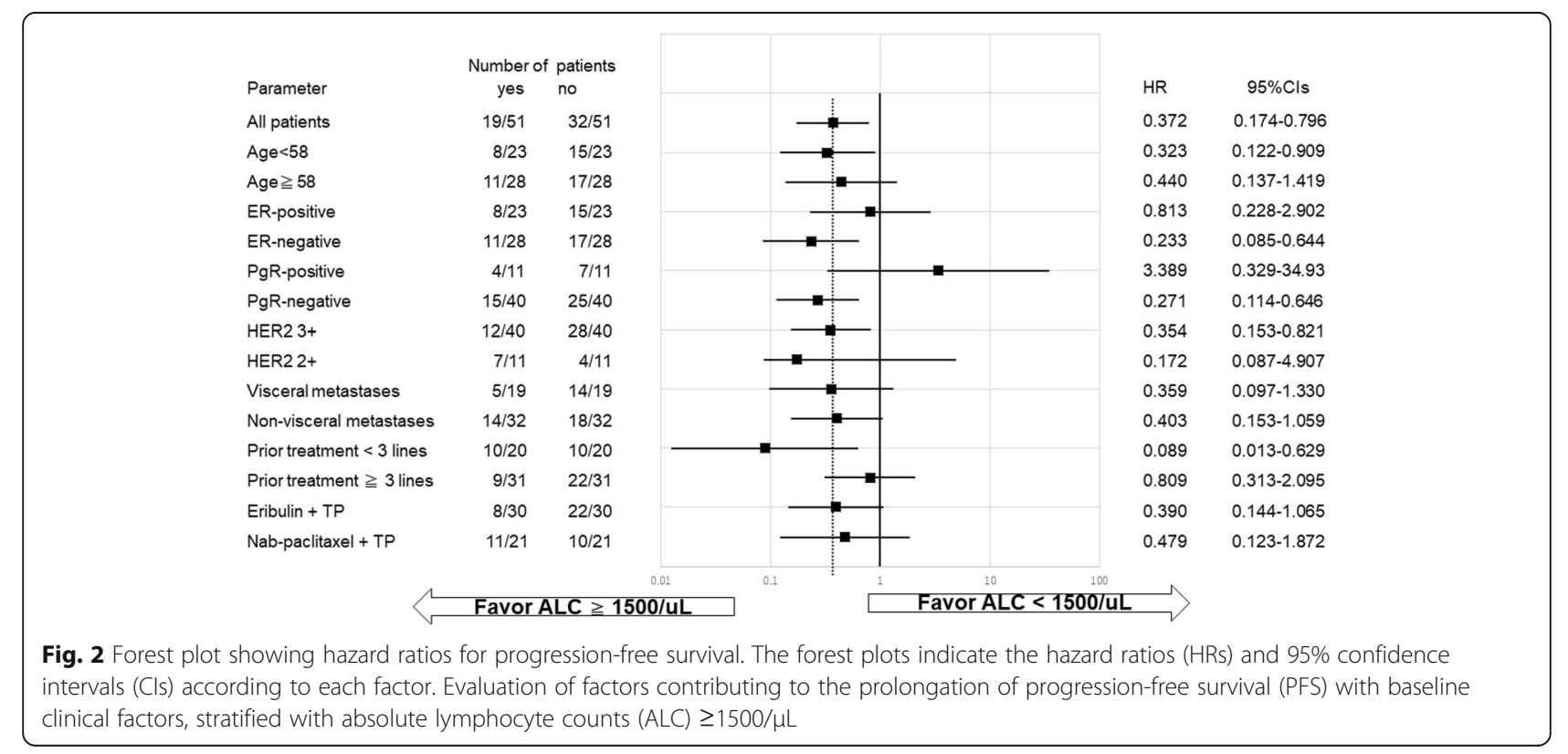


Table 3 Comparison of baseline patients' characteristics based on patients with baseline absolute lymphocyte counts

\begin{tabular}{|c|c|c|c|}
\hline \multirow[t]{2}{*}{ Characteristics } & \multicolumn{2}{|c|}{ Absolute lymphocyte counts } & \multirow[t]{2}{*}{$P$ value } \\
\hline & $\begin{array}{l}\geq 1500 / \mu \mathrm{L} \\
(n=19)\end{array}$ & $\begin{array}{l}<1500 / \mu \mathrm{L} \\
(n=32)\end{array}$ & \\
\hline Median age (range) & $58(32-76)$ yo & $58(31-77)$ yo & 0.7109 \\
\hline \multicolumn{4}{|l|}{ Estrogen receptor } \\
\hline Positive & $8(42 \%)$ & 15 (47\%) & \multirow[t]{2}{*}{0.7787} \\
\hline Negative & $11(58 \%)$ & 17 (53\%) & \\
\hline \multicolumn{4}{|l|}{ Progesterone receptor } \\
\hline Positive & $4(21 \%)$ & $7(22 \%)$ & \multirow[t]{2}{*}{1.0000} \\
\hline Negative & $15(79 \%)$ & $25(78 \%)$ & \\
\hline \multicolumn{4}{|l|}{ HER2 } \\
\hline $3+$ & $12(63 \%)$ & $28(88 \%)$ & \multirow[t]{2}{*}{0.0754} \\
\hline 2+ FISH amplification & $7(37 \%)$ & $4(12 \%)$ & \\
\hline Visceral metastases & $5(26 \%)$ & $14(47 \%)$ & \multirow[t]{2}{*}{0.2465} \\
\hline Non-visceral metastases & $14(74 \%)$ & $18(56 \%)$ & \\
\hline \multicolumn{4}{|l|}{ Prior treatment } \\
\hline$<3$ lines & $13(68 \%)$ & $17(53 \%)$ & \multirow[t]{2}{*}{0.3808} \\
\hline$\geq 3$ lines & $6(32 \%)$ & $15(47 \%)$ & \\
\hline \multicolumn{4}{|c|}{$\begin{array}{l}\text { Combination chemotherapy } \\
\text { with trastuzumab plus } \\
\text { pertuzumab }\end{array}$} \\
\hline Eribulin & $8(42 \%)$ & $22(69 \%)$ & \multirow[t]{2}{*}{0.0815} \\
\hline Nab-paclitaxel & $11(58 \%)$ & 10 (31\%) & \\
\hline
\end{tabular}

patients treated with PT in combination with other chemotherapies or endocrine therapies.

This study has several limitations. First, we used a retrospective design from two single prospective phase 2 studies with relatively small sample sizes. Second, prior chemotherapies may affect baseline levels of PBBPs. However, because a positive association between ALC levels and PFS was consistently recognized in patients with and without prior chemotherapy, the influence of prior chemotherapy appears to be limited. There was no significant difference for any PBBP during the course of treatment (data not shown), but the prognostic significance of PBBPs during the treatment course is currently unknown. Considering these limitations, prospective studies that include a large number of patients are needed to further elucidate the results of the present study.

\section{Conclusions}

Our findings strongly suggest that ALC at baseline is a useful predictor for prolonged PFS in HER2-positive $\mathrm{ABC}$ patients treated with $\mathrm{PT}$ in combination with ERI or Nab-PTX. Baseline ALC may be indicative of pre-existing anti-tumor host immunity or the potential immune response following PT-based therapy. Future research might focus on the baseline systemic immune response evaluable by ALC in daily clinical practice.

\section{Abbreviations}

ABC: Advanced breast cancer; ADCC: Antibody-dependent cell-mediated cytotoxicity; ALC: Absolute lymphocyte count; Cl: Confidence interval; CLEOPATRA: The investigators in the Clinical Evaluation of Pertuzumab and Trastuzumab; ECOG-PS: Eastern Cooperative Oncology Group Performance Status; EGFR: Epidermal growth factor receptor; ER: Estrogen receptor; ERI: Eribulin; HER2: Human epidermal growth factor receptor 2; HR: Hazard ratio; IHC: Immunohistochemistry; JSPS: Japan Society for Promotion of Science; Nab-PTX: Nab-paclitaxel; NL: Natural killer; NLR: Neutrophil to lymphocyte ratio; PBBPs: Peripheral blood-based parameters;

PFS: Progression-free survival; PgR: Progesterone receptor; PLR: Platelet to lymphocyte ratio; PT: Pertuzumab and trastuzumab; TILs: Tumor-infiltrating lymphocytes

\section{Acknowledgments}

The authors would like to thank the patients enrolled in this study, and their families.

\section{Funding}

This work was supported by the Japan Society for the Promotion of Science (JSPS) KAKENHI Grant Number JP16K10485.

Availability of data and materials

The dataset of the current study were available from the corresponding author on reasonable request.

\section{Authors' contributions}

All authors made substantial contributions to one or more of the following: the study conception and design (KA, YaM); acquisition of data (KA, YI, IF, KK, YoM, MI, AK, YT, CE, HS, SO) or analysis (KA, YaM); and interpretation of data (KA, YaM). KA and YaM drafted the article and all other authors contributed to revising the article critically for important intellectual content. All authors read and approved the final manuscript.

\section{Ethics approval and consent to participate}

The study was conducted in accordance with the guidelines for Good Clinical Practice and the Declaration of Helsinki. Written informed consent was obtained from each participant before undertaking any study-related procedures. This study was approved by the ethics committee of The Cancer Institute Hospital of the Japanese Foundation for Cancer Research (\#2013-1096 and \#2017-1076) and the Hyogo College of Medicine (No. 1061 and No. 2720).

\section{Consent for publication}

Not applicable.

\section{Competing interests}

$K$ Araki has performed contracted research for Eisai, Novartis, and AstraZeneca. Y Ito has performed contracted research for Chugai, Parexel, and Eisai. S Ohno has performed contracted research for Chugai and AstraZeneca. Y Miyoshi has received research funding and honoraria from Eisai Co., Ltd., Chugai Pharmaceutical Co., Ltd., and TAIHO PHARMACEUTICAL Co., Ltd., AstraZeneca plc, Novartis Pharma K.K., Pfizer Japan Inc., MSD K.K. Eli Lilly Japan K.K., Kyowa Hakko Kirin Co., Ltd., and DAIICHI SANKYO Co., Ltd. The authors have no other relevant affiliations or financial involvement with any organization or entity with a financial interest in or financial conflict with the subject matter or materials discussed in the manuscript apart from those disclosed.

\section{Publisher's Note}

Springer Nature remains neutral with regard to jurisdictional claims in published maps and institutional affiliations. 


\section{Author details}

'Hyogo College of Medicine, Department of Surgery, Division of Breast and Endocrine Surgery, 1-1 Mukogawa, Nishinomiya, Hyogo 663-8501, Japan. ${ }^{2}$ The Cancer Institute Hospital of the Japanese Foundation for Cancer Research, Breast Medical Oncology, 3-8-31 Ariake, Koto-ku, Tokyo 135-8550, Japan. ${ }^{3}$ Kansai Rosai Hospital, Department of Surgery, 3-1-69 Inabaso, Amagasaki, Hyogo 660-8511, Japan. ${ }^{4}$ Hyogo Prefectural Amagasaki General Medical Center, Department of Breast Surgery, 2-17-77 East Namba-machi, Amagasaki, Hyogo 660-8550, Japan.

Received: 9 April 2018 Accepted: 2 October 2018

Published online: 16 October 2018

\section{References}

1. Martin M, Lopez-Tarruella S. Emerging therapeutic options for HER2-positive breast Cancer. Am Soc Clin Oncol Educ Book/ASCO Am Soc Clin Oncol Meet. 2016;35:e64-70.

2. Swain SM, Baselga J, Kim SB, Ro J, Semiglazov V, Campone M, Ciruelos E, Ferrero JM, Schneeweiss A, Heeson S, et al. Pertuzumab, trastuzumab, and docetaxel in HER2-positive metastatic breast cancer. N Engl J Med. 2015;372(8):724-34.

3. Araki K, Fukada I, Yanagi H, Kobayashi K, Shibayama T, Horii R, Takahashi S, Akiyama F, Ohno S, Ito Y. First report of eribulin in combination with pertuzumab and trastuzumab for advanced HER2-positive breast cancer. Breast. 2017:35:78-84.

4. Muntasell A, Cabo M, Servitja S, Tusquets I, Martinez-Garcia M, Rovira A, Rojo F, Albanell J, Lopez-Botet M. Interplay between natural killer cells and anti-HER2 antibodies: perspectives for breast Cancer immunotherapy. Front Immunol. 2017;8:1544.

5. Lee SC, Srivastava RM, Lopez-Albaitero A, Ferrone S, Ferris RL. Natural killer (NK): dendritic cell (DC) cross talk induced by therapeutic monoclonal antibody triggers tumor antigen-specific $T$ cell immunity. Immunol Res. 2011;50(2-3):248-54.

6. Luen SJ, Salgado R, Fox S, Savas P, Eng-Wong J, Clark E, Kiermaier A, Swain SM, Baselga J, Michiels S, et al. Tumour-infiltrating lymphocytes in advanced HER2-positive breast cancer treated with pertuzumab or placebo in addition to trastuzumab and docetaxel: a retrospective analysis of the CLEOPATRA study. Lancet Oncol. 2017:18(1):52-62

7. Schreiber RD, Old LJ, Smyth MJ. Cancer immunoediting: integrating immunity's roles in cancer suppression and promotion. Science. 2011;331(6024):1565-70.

8. Chen DS, Mellman I. Oncology meets immunology: the cancer-immunity cycle. Immunity. 2013;39(1):1-10.

9. Slaney CY, Kershaw MH, Darcy PK. Trafficking of T cells into tumors. Cancer Res. 2014;74(24):7168-74.

10. Gros A, Parkhurst MR, Tran E, Pasetto A, Robbins PF, Ilyas S, Prickett TD, Gartner JJ, Crystal JS, Roberts IM, et al. Prospective identification of neoantigen-specific lymphocytes in the peripheral blood of melanoma patients. Nat Med. 2016:22(4):433-8.

11. Ray-Coquard I, Cropet C, Van Glabbeke M, Sebban C, Le Cesne A, Judson I, Tredan O, Verweij J, Biron P, Labidi I, et al. Lymphopenia as a prognostic factor for overall survival in advanced carcinomas, sarcomas, and lymphomas. Cancer Res. 2009;69(13):5383-91.

12. Koh CH, Bhoo-Pathy N, Ng KL, Jabir RS, Tan GH, See MH, Jamaris S, Taib NA. Utility of pre-treatment neutrophil-lymphocyte ratio and platelet-lymphocyte ratio as prognostic factors in breast cancer. Br J Cancer. 2015;113(1):150-8.

13. Asano Y, Kashiwagi S, Onoda N, Noda S, Kawajiri H, Takashima T, Ohsawa M, Kitagawa S, Hirakawa K. Predictive value of neutrophil/lymphocyte ratio for efficacy of preoperative chemotherapy in triple-negative breast Cancer. Ann Surg Oncol. 2016;23(4):1104-10.

14. Xu J, Ni C, Ma C, Zhang L, Jing X, Li C, Liu Y, Qu X. Association of neutrophil/lymphocyte ratio and platelet/lymphocyte ratio with ER and PR in breast cancer patients and their changes after neoadjuvant chemotherapy. Clin Transl Oncol. 2017;19(8):989-96.

15. Chen Y, Chen K, Xiao X, Nie Y, Qu S, Gong C, Su F, Song E. Pretreatment neutrophil-to-lymphocyte ratio is correlated with response to neoadjuvant chemotherapy as an independent prognostic indicator in breast cancer patients: a retrospective study. BMC Cancer. 2016;16:320.

16. Rodríguez DM, Guerrero ME, Maldonado BM, Vollbracht C, Herrera SA. Total lymphocyte count in cancer patients with lymphopenia treated with intravenous vitamin C: Results of an observational study. Translat Med Commun. 2017;2:3
17. Efremova M, Finotello F, Rieder D, Trajanoski Z. Neoantigens generated by individual mutations and their role in Cancer immunity and immunotherapy. Front Immunol. 2017;8:1679.

18. Kroemer G, Senovilla L, Galluzzi L, Andre F, Zitvogel L. Natural and therapyinduced immunosurveillance in breast cancer. Nat Med. 2015;21(10):1128-38.

19. Scheuer W, Friess T, Burtscher H, Bossenmaier B, Endl J, Hasmann M. Strongly enhanced antitumor activity of trastuzumab and pertuzumab combination treatment on HER2-positive human xenograft tumor models. Cancer Res. 2009;69(24):9330-6.

20. Watanabe T, Hida Al, Inoue N, Imamura M, Fujimoto Y, Akazawa K, Hirota S, Miyoshi Y. Abundant tumor infiltrating lymphocytes after primary systemic chemotherapy predicts poor prognosis in estrogen receptor-positive/HER2negative breast cancers. Breast Cancer Res Treat. 2018;168(1):135-45.

21. Galluzzi L, Buque A, Kepp O, Zitvogel L, Kroemer G. Immunological effects of conventional chemotherapy and targeted anticancer agents. Cancer Cell. 2015;28(6):690-714.

22. Kavallaris M. Microtubules and resistance to tubulin-binding agents. Nat Rev Cancer. 2010;10(3):194-204.

\section{Ready to submit your research? Choose BMC and benefit from:}

- fast, convenient online submission

- thorough peer review by experienced researchers in your field

- rapid publication on acceptance

- support for research data, including large and complex data types

- gold Open Access which fosters wider collaboration and increased citations

- maximum visibility for your research: over $100 \mathrm{M}$ website views per year

At BMC, research is always in progress.

Learn more biomedcentral.com/submissions 